\title{
Is kidney function affecting the management of myocardial infarction? A retrospective cohort study in patients with normal kidney function, chronic kidney disease stage III-V, and ESRD
}

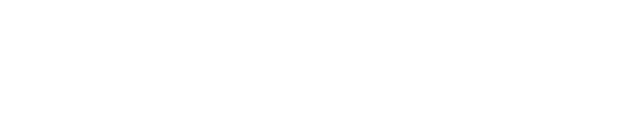

Marc Saad'

Boutros Karam'

Geovani Faddoul ${ }^{2}$

Youssef El Douaihy'

Harout Yacoub'

Hassan Baydoun ${ }^{3}$

Christine Boumitri'

Iskandar Barakat'

Chadi Saifan ${ }^{4}$

Elie El-Charabaty ${ }^{4}$

Suzanne El Sayegh ${ }^{4}$

'Department of Internal Medicine, Staten Island University Hospital, Staten Island, ${ }^{2}$ Department of Nephrology, Icahn School of Medicine, New York, NY, ${ }^{3}$ Department of Cardiology, Tulane University Medical Center, New Orleans, LA, ${ }^{4}$ Department of Nephrology, Staten Island University Hospital, Staten Island, NY, USA
Correspondence: Marc Saad Department of Internal Medicine, Staten Island University Hospital, Department of Internal Medicine, Staten Island University Hospital, 475 Seaview Avenue, Staten Island, NY 10305, USA

Emailmarcsaadmd@gmail.com
Abstract: Patients with chronic kidney disease (CKD) are three times more likely to have myocardial infarction (MI) and suffer from increased morbidity and higher mortality. Traditional and unique risk factors are prevalent and constitute challenges for the standard of care. However, CKD patients have been largely excluded from clinical trials and little evidence is available to guide evidence-based treatment of coronary artery disease in patients with CKD. Our objective was to assess whether a difference exists in the management of MI (ST-segment elevation myocardial infarction and non-ST-segment elevation myocardial infarction) among patients with normal kidney function, CKD stage III-V, and end-stage renal disease (ESRD) patients. We conducted a retrospective cohort study on patients admitted to Staten Island University Hospital for the diagnosis of MI between January 2005 and December 2012. Patients were assigned to one of three groups according to their kidney function: Data collected on the medical management and the use of statins, platelet inhibitors, beta-blockers, and angiotensin converting enzyme inhibitors/angiotensin receptor blockers were compared among the three cohorts, as well as medical interventions including: catheterization and coronary artery bypass graft (CABG) when indicated. Chi-square test was used to compare the proportions between nominal variables. Binary logistic analysis was used in order to determine associations between treatment modalities and comorbidities, and to account for possible confounding factors. Three hundred and thirty-four patients (mean age 67.2 \pm 13.9 years) were included. In terms of management, medical treatment was not different among the three groups. However, cardiac catheterization was performed less in ESRD when compared with no CKD and CKD stage III-V (45.6\% vs 74\% and 93.9\%) $(P<0.001)$. CABG was performed in comparable proportions in the three groups and $\mathrm{CABG}$ was not associated with the degree of CKD $(P=0.078)$ in binary logistics regression. Cardiac catheterization on the other hand carried the strongest association among all studied variables $(P<0.001)$. This association was maintained after adjusting for other comorbidities. The length of stay for the three cohorts (non-CKD, CKD stage III-V, and ESRD on hemodialysis) was 16,17 , and 15 days, respectively and was not statistically different. Many observations have reported discrimination of care for patients with CKD considered suboptimal candidates for aggressive management of their cardiac disease. In our study, medical therapy was achieved at high percentage and was comparable among groups of different kidney function. However, kidney disease seems to affect the management of patients with acute MI; percutaneous coronary angiography is not uniformly performed in patients with CKD and ESRD when compared with patients with normal kidney function.

Keywords: myocardial infarction, chronic kidney disease, end-stage renal disease 


\section{Introduction}

Ischemic heart disease is the most common cause of death in patients with chronic kidney disease (CKD). CKD in acute coronary syndrome (ACS) is independently associated with increased morbidity and mortality. ${ }^{1}$ Traditional and unique risk factors are prevalent putting patients with CKD at higher risk of developing coronary artery disease (CAD) and constitute challenges for the standard of care. ${ }^{1}$ Despite recognizing this high-risk group, patients with CKD have been largely excluded from randomized controlled trials and management guidelines are not established. ${ }^{1}$

ACS diagnosis is based on the clinical presentation of ischemic symptoms, cardiac biomarkers, and electrocardiogram changes. Compared with general population, CKD patients commonly have atypical presentation as in elderly and diabetic; in addition, diagnostic markers have low predictive value since many patients have elevated troponins without having ACS. ${ }^{1,2}$

In fact, CKD patients have better outcomes when evidencebased therapy is adopted. ${ }^{2}$ Analysis of data from large clinical trials demonstrated that the implementation of invasive treatment is associated with better prognosis in patients with endstage renal disease (ESRD) and moderate CKD. However, one study demonstrated that patients with ACS and low glomerular filtration rate (GFR) are less likely to receive invasive interventions, and if they received angiography, they may not undergo revascularization., ${ }^{3,4}$ It is not clear if these strategies would have similar risk-benefit profiles in the treatment of renal impaired patients. For example, in a review of randomized trials, antiplatelet therapy in patients with CKD had no significant reduction of cardiovascular events or death but had increased risk of major bleeding. ${ }^{5}$ The risk of complications increases with the reduction in GFR, and patients on dialysis have the worst prognosis. ${ }^{6}$

Optimal therapy is yet to be defined. As such, ACS in CKD remains a challenge for the cardiologist. Significant data regarding inpatient care of patients with CKD and ESRD presenting with ACS come from Medicare and Medicare data reports available from the United States Renal Data System, which reported significant disparities in care in patients with CKD and ESRD. ${ }^{7}$ Another report from the National Cardiovascular Data Acute Coronary Treatment and Intervention Outcomes Network Registry also reported that these disparities exist. ${ }^{8}$

The aim of this study was to evaluate differences in inpatient care among patients without CKD, patients with CKD stage III-V, and patients with ESRD requiring renal replacement therapy admitted to our institution with myocardial infarction (MI).

\section{Materials and methods Design and methodology}

This was a retrospective study including all patients admitted to Staten Island University Hospital with a diagnosis of MI between January 2005 and December 2012. Patients were excluded from the study if they had a diagnosis of acute kidney injury (AKI) at the time of presentation or had developed AKI during hospitalization but no CKD was present at the time of admission. The study was approved by the North Shore-LIJ Review Board. We identified these differences by reviewing medical records.

Patients were stratified into groups based on kidney function on admission, by estimating GFR using the Modification of Diet in Renal Disease equation, which uses the serum creatinine level at the time of presentation. It is defined as normal (GFR $\geq 60 \mathrm{~mL} / \mathrm{min} / 1.73 \mathrm{~m}^{2}$ ), moderate CKD (GFR 30-59 $\left.\mathrm{mL} / \mathrm{min} / 1.73 \mathrm{~m}^{2}\right)$, severe CKD $\left(\mathrm{GFR}<30 \mathrm{~mL} / \mathrm{min} / 1.73 \mathrm{~m}^{2}\right)$, and ESRD patients requiring dialysis. We hypothesized that there is no difference among the three groups of patients with respect to medical therapy and invasive cardiac procedures (such as cardiac catheterization and/or coronary artery bypass graft [CABG], if indicated), highlighting the need to apply optimal treatment strategies that high-risk patients would benefit from.

\section{Statistical analysis}

The primary outcome variables were length of stay, invasive cardiac procedures (such as cardiac catheterization and/or $\mathrm{CABG}$, if indicated), and use of medication.

For the analysis of length of stay, an analysis of covariance model comprised the fixed, categorical effects of the three groups of patients to be used, adjusting for potential confounding factors such as sex, age, race, and smoking status. Chi-square test was used to compare the proportions between nominal variables. Binary logistic analysis was used in order to determine associations between treatment modalities and comorbidities, and to account for possible confounding factors.

All statistical tests of significance were two-sided and were conducted at the 0.05 level of significance. All statistical analyses were conducted in SAS (Version 9.3, SAS Institute Inc., Cary, NC, USA).

\section{Results}

\section{Patient demographics and clinical characteristics}

Three hundred and thirty-four patients were included, out of which $68.6 \%$ were males (mean age $67.2 \pm 13.9$ years), 
76\% were Caucasian, 8.1\% were African American, and $15.9 \%$ were Hispanic and other. One hundred and sixty-eight patients $(50.3 \%)$ had ST-segment elevation MI, while 166 patients $(49.7 \%$ ) had non-ST-segment elevation MI; 39.2\% had GFR $>60 \mathrm{~mL} / \mathrm{min} / 1.73 \mathrm{~m}^{2}$ on admission, $35.6 \%$ had CKD stage III-V, and 24\% had ESRD on dialysis; $44.6 \%$ had diabetes, $73.1 \%$ had hypertension (HTN), 55.1\% had dyslipidemia (DL), and $27.5 \%$ had congestive heart failure (CHF) (Table 1).

The length of stay for three cohorts (non-CKD, CKD stage III-V, and ESRD on hemodialysis [HD]) was 16, 17, and 15 days, respectively and was not statistically different.

In terms of medical management, treatment with aspirin, platelet inhibitors, angiotensin converting enzyme inhibitors, angiotensin receptor blockers, beta-blockers, and statins was achieved at high rates in our study and was similar irrespective of kidney function (Table 2).

However, coronary angiography was less likely to be performed in patients with ESRD compared with non-CKD (45.6\% vs 93.9\%) $(P<0.001)$. CABG was performed in $14.4 \%$ of non-CKD patients, $24.0 \%$ in CKD stage III-V, and $13.6 \%$ of patients on dialysis. It did not differ among the three groups $(P=0.07)$. To note, ESRD and CKD stage III-V were more

Table I Descriptive analysis

\begin{tabular}{|c|c|}
\hline Variable & $\begin{array}{l}\text { Frequency }(\%) \\
\text { ( } \pm \text { standard deviation) }\end{array}$ \\
\hline Age & $* 67.2( \pm 13.9)$ \\
\hline \multicolumn{2}{|l|}{ Race } \\
\hline Caucasian & $254(76.05)$ \\
\hline African American & $27(8.08)$ \\
\hline Hispanic & $3(0.9)$ \\
\hline Other & $50(14.97)$ \\
\hline \multicolumn{2}{|l|}{ Sex } \\
\hline Female & $105(31.4)$ \\
\hline Male & $229(68.6)$ \\
\hline \multicolumn{2}{|l|}{ ACS } \\
\hline NSTEMI & $166(49.7)$ \\
\hline STEMI & $168(50.3)$ \\
\hline \multicolumn{2}{|l|}{ Kidney disease } \\
\hline Non-CKD (eGFR >60), $\mathrm{mL} / \mathrm{min} / \mathrm{l} .73 \mathrm{~m}^{2}$ & 131 (39.2) \\
\hline $\begin{array}{l}\text { CKD stage III-V (eGFR }<60), \mathrm{mL} / \\
\mathrm{min} / 1.73 \mathrm{~m}^{2}\end{array}$ & $119(35.6)$ \\
\hline ESRD on HD & $80(24.0)$ \\
\hline Diabetes (type 2) & $149(44.6)$ \\
\hline Hypertension & $244(73.1)$ \\
\hline Hypercholesterolemia & $184(55.1)$ \\
\hline $\mathrm{CHF}$ & $92(27.5)$ \\
\hline
\end{tabular}

Note: *Data presented as mean ( \pm standard deviation).

Abbreviations: ACS, acute coronary syndrome; NSTEMI, non-ST-segment elevation myocardial infarction; STEMI, ST-segment elevation myocardial infarction; CKD, chronic kidney disease; eGFR, estimated glomerular filtration rate; ESRD, endstage renal disease; HD, hemodialysis; CHF, congestive heart failure. likely to go to $\mathrm{CABG}$ when catheterization was performed (30\%) when compared with non-CKD patients (15\%).

In binary logistics regression, medical therapy was not associated with a particular comorbidity with the exceptions of beta-blockers with HTN $(P=0.020)$ and statins with CHF and DL ( $P=0.018$ and 0.025 , respectively). CABG was not associated with degree of CKD $(P=0.074)$ (HTN, diabetes mellitus, and CHF showed a stronger association). Cardiac catheterization on the other hand carried the strongest association among all studied variables $(P<0.001)$. This association was maintained after adjusting for other comorbidities.

\section{Discussion}

Patients with CKD and/or ESRD have a higher risk of CAD and morbidity/mortality from CAD, and should ideally receive current standard of care, as do patients with normal renal function. However, detailed reports including length of stay, differences in utilization of current evidence-based medical management, and invasive coronary intervention including coronary bypass surgery are not available on CKD and ESRD patients regarding CAD treatment. ${ }^{9}$

Many observations have reported discrimination of care for patients with CKD; studies showed that CKD patients were less likely to receive evidence-based therapy in ACS compared with patients without $\mathrm{CKD}$ as they are considered suboptimal candidates for aggressive management of their cardiac disease. ${ }^{2,9}$

In our study, the exclusion criterion of AKI regardless of the presence of prior CKD was used to eliminate bias regarding the administration or withholding medication, intervention, and the length of stay. Medical therapy was achieved in high percentage in the three cohorts (non-CKD, CKD stage III-V, and ESRD on HD) and was comparable regardless of kidney function (Figure 1).

As this study was observational and limited itself to the timeframe of hospitalization, we could only measure adherence to prescribing optimal therapy. A follow-up of the patients would shed the light on the complications related to therapy itself, which is beyond the scope of this study.

Percutaneous coronary revascularization improves long-term survival when compared with medical therapy, ${ }^{9}$ yet we found that ESRD patients on HD were getting less catheterization than the other two groups ( $45.6 \%$ vs $75.8 \%$ and $93.9 \%)(P<0.001)$. Unique risk factors were present in these patients putting them at once at high risk of CAD and of procedural complications.

The Enoxaparin and Thrombolysis Reperfusion for Acute Myocardial Infarction Treatment, Thrombolysis in 
Table 2 Population characteristics, medications, and interventions distribution

\begin{tabular}{|c|c|c|c|c|}
\hline & Non-CKD $($ eGFR $>60)$ & $\begin{array}{l}\text { CKD stage III-V } \\
(\text { eGFR }<60), \mathrm{mL} / \mathrm{min} / 1.73 \mathrm{~m}^{2}\end{array}$ & ESRD on HD & Significance $\left(\chi^{2}\right)$ \\
\hline $\mathrm{CHF}$ & 15 (1 I.4\%) & 49 (39.8\%) & $30(37.0 \%)$ & 0.000 \\
\hline Hyperlipidemia & 68 (51.5\%) & $76(61.8 \%)$ & $42(51.2 \%)$ & 0.182 \\
\hline Hypertension & $70(53.0 \%)$ & $105(85.4 \%)$ & 72 (87.8\%) & $<0.00 \mathrm{I}$ \\
\hline Diabetes & $33(25.0 \%)$ & $64(51.6 \%)$ & $52(63.4 \%)$ & $<0.001$ \\
\hline Race & & & & 0.003 \\
\hline Caucasian & 95 (73.6\%) & $106(86.9 \%)$ & $57(72.2 \%)$ & \\
\hline African American & $16(12.4 \%)$ & $4(3.3 \%)$ & $6(7.6 \%)$ & \\
\hline Hispanic & $0(0.0 \%)$ & $0(0.0 \%)$ & $3(3.8 \%)$ & \\
\hline Other & $18(14.0 \%)$ & $12(9.8 \%)$ & $13(16.5 \%)$ & \\
\hline CABG & 19 (14.4\%) & $29(24.0 \%)$ & II (I3.6\%) & 0.074 \\
\hline Cardiac catheterization & 124 (93.9\%) & 91 (75.8\%) & $36(45.6 \%)$ & $<0.00 \mathrm{I}$ \\
\hline ASA & $122(96.1 \%)$ & 90 (90.9\%) & 67 (90.5\%) & 0.204 \\
\hline Beta-blockers & 115 (90.6\%) & 89 (89.9\%) & 69 (93.2\%) & 0.729 \\
\hline Platelet inhibitors & 109 (86.5\%) & $76(77.6 \%)$ & $54(74.0 \%)$ & 0.067 \\
\hline ACE inhibitors/ARB & $70(55.1 \%)$ & $47(47.5 \%)$ & $32(43.2 \%)$ & 0.232 \\
\hline Statins & 121 (95.3\%) & $83(83.8 \%)$ & $65(87.8 \%)$ & 0.017 \\
\hline
\end{tabular}

Abbreviations: CKD, chronic kidney disease; eGFR, estimated glomerular filtration rate; ESRD, end-stage renal disease; HD, hemodialysis; CHF, congestive heart failure; $C A B G$, coronary artery bypass graft; ASA, aspirin; $A C E$, angiotensin converting enzyme; $A R B$, angiotensin receptor blockers.

Myocardial Infarction-Study 25 substudy demonstrated that bleeding is increased by $50 \%$ for every $30 \mathrm{~mL} / \mathrm{min}$ decreased in GFR. ${ }^{1}$ CKD/ESRD patients are known to have platelet dysfunction leading to prolonged bleeding time, which can complicate invasive procedure in patients with ACS. Another consideration is the altered drug metabolism in patients with ESRD. There is a required dose adjustment in patients with CKD due to higher risk of medication-related adverse events. Although anticoagulant drugs are the mainstay of ACS management, their benefit is still retained in CKD patients, and they should be used with caution to prevent bleeding complications.

Contrast-induced nephropathy (CIN) is a well-known complication after cardiac catheterization. Patients with $\mathrm{CKD}$ are at higher risk for $\mathrm{CIN}$ from iodinated radiocontrast used for coronary angiography ${ }^{10}$ and the risk is inversely proportional to GFR. ${ }^{2}$ Volume expansion with normal saline was shown to be the most effective strategy to prevent and reduce CIN.

Moreover, ESRD patients have extended small vessel disease that we believe is frequently responsible for their ischemic heart disease. Thus, there will be less interventions to fix distal small lesion within calcified vessel. In addition, ESRD will frequently have type 2 MI (supply/demand myocardial infarction) in a setting of significant deconditioning precipitated by prevalent comorbidities.

It is due to those risks and other reasons that patients with CKD may receive less percutaneous coronary interventions (PCIs) and thus a less invasive treatment. Complications may lead to prolonged hospital stay and higher hospital mortality. ${ }^{9}$

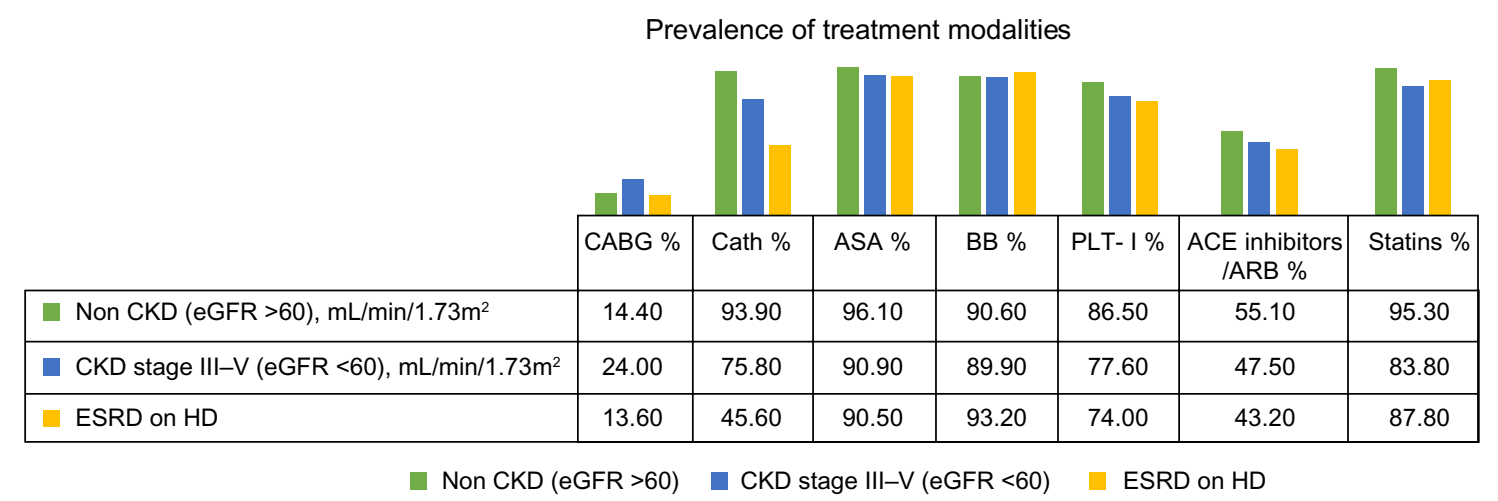

Figure I Outcome and interventions analysis.

Abbreviations: CKD, chronic kidney disease; eGFR, estimated glomerular filtration rate; CABG, coronary artery bypass graft; Cath, catheterization; ASA, aspirin; BB, betablockers; PLT-I, platelet inhibitors; ACE, angiotensin converting enzyme; ARB, angiotensin receptor blockers; ESRD, end-stage renal disease; HD, hemodialysis. 
CABG was performed in comparable percentage among the three cohorts; furthermore, $\mathrm{CABG}$ was more likely to be done when catheterization was performed in ESRD patients on HD.

No evidence-based guidelines are available to direct the care for patients on $\mathrm{HD} .{ }^{9}$ Either CABG surgery or PCI can be used to treat multivessel CAD. Treatment modalities are mostly based on experiences and opinions rather than evidences. ${ }^{11}$

Existing studies report a significantly higher postoperative morbidity (cardiovascular complications and rate of permanent HD) and early mortality among patients with CKD who underwent $\mathrm{CABG}$ when compared with patients with preserved kidney function. ${ }^{11,12}$

When CABG and PCI were compared in CKD, observational studies reported conflicting results. While some observational studies reported long-term CABG mortality benefit over PCI despite a higher early risk of death and ESRD, other studies suggested comparable outcomes. ${ }^{13-15} \mathrm{In}$ a recent study, ESRD patients on HD with multivessel and/or left main disease had reduced risk of cardiac death, sudden death, and MI and any revascularization in 5-year follow-up when they underwent CABG compared with PCI. However, the risk of all-cause death was not different. ${ }^{12,16,17}$

Physicians are overcoming challenges based on individual cases. The current challenge is to study the CAD patient with CKD in prospective randomized trials to provide an evidence-based approach to therapy. In the absence of such information, aggressive control of CAD risk factors and timely intervention for symptomatic CAD is suggested. ${ }^{9}$

\section{Conclusion}

In conclusion, there is no difference among the three groups of patients (patients without CKD, patients with CKD, and patients with ESRD) with respect to medical management. On the other hand, catheterization was achieved less in ESRD on HD when compared with non-CKD patients. These observations will help us to evaluate the reasons for these disparities. Furthermore, we hope to identify barriers to the current standard of care for CAD provided to the patients with CKD and ESRD.

\section{Disclosure}

The authors report no conflicts of interest in this work.

\section{References}

1. Franczyk-Skóra B, Gluba A, Banach M, Rysz J. Treatment of nonST-elevation myocardial infarction and ST-elevation myocardial infarction in patients with chronic kidney disease. Arch Med Sci. 2013;9(6):1019-1027.
2. Roberts JK, McCullough PA. The management of acute coronary syndromes in patients with chronic kidney disease. Adv Chronic Kidney Dis. 2014;21(6):472-479.

3. Wong JA, Goodman SG, Yan RT, et al; Canadian Acute Coronary Syndromes I and II, and Canadian Global Registry of Acute Coronary Events (GRACE/GRACE) Investigators. Temporal management patterns and outcomes of non-ST elevation acute coronary syndromes in patients with kidney dysfunction. Eur Heart J. 2009;30(5):549-557.

4. Hanna EB, Chen AY, Roe MT, Saucedo JF. Characteristics and in-hospital outcomes of patients presenting with non-ST-segment elevation myocardial infarction found to have significant coronary artery disease on coronary angiography and managed medically: stratification according to renal function. Am Heart J. 2012;164(1):52-57.

5. Palmer SC, Di Micco L, Razavian M, et al. Effects of antiplatelet therapy on mortality and cardiovascular and bleeding outcomes in persons with chronic kidney disease: a systematic review and meta-analysis. Ann Intern Med. 2012;156(6):445-459.

6. Rhee JW, Wiviott SD, Scirica BM, et al. Clinical features, use of evidence-based therapies, and cardiovascular outcomes among patients with chronic kidney disease following non-ST-elevation acute coronary syndrome. Clin Cardiol. 2014;37(6):350-356.

7. Collins AJ, Foley RN, Herzog C, et al. Excerpts from the US Renal Data System 2009 Annual Data Report. Am J Kidney Dis. 2010;55(1 Suppl 1): S1-S420, A426-A427.

8. Fox CS, Muntner P, Chen AY, et al; Acute Coronary Treatment and Intervention Outcomes Network registry. Use of evidence-based therapies in short-term outcomes of ST-segment elevation myocardial infarction and non-ST-segment elevation myocardial infarction in patients with chronic kidney disease: a report from the National Cardiovascular Data Acute Coronary Treatment and Intervention Outcomes Network registry. Circulation. 2010;121(3):357-365.

9. Marenzi G, Cosentino N, Guastoni C. How to balance risks and benefits in the management of CKD patients with coronary artery disease. J Nephrol. 2015;28(4):403-413.

10. Mehran R, Aymong ED, Nikolsky E, et al. A simple risk score for prediction of contrast-induced nephropathy after percutaneous coronary intervention: development and initial validation. $J$ Am Coll Cardiol. 2004;44(7):1393-1399.

11. Ashrith G, Lee VV, Elayda MA, Reul RM, Wilson JM. Short- and long-term outcomes of coronary artery bypass grafting or drug-eluting stent implantation for multivessel coronary artery disease in patients with chronic kidney disease. Am J Cardiol. 2010;106(3):348-353.

12. Marui A, Kimura T, Nishiwaki N, et al; CREDO-Kyoto PCI/CABG Registry Cohort-2 Investigators. Percutaneous coronary intervention versus coronary artery bypass grafting in patients with end-stage renal disease requiring dialysis (5-year outcomes of the CREDO-Kyoto PCI/ CABG Registry Cohort-2). Am J Cardiol. 2014;114(4):555-561.

13. Shroff GR, Solid CA, Herzog CA. Long-term survival and repeat coronary revascularization in dialysis patients after surgical and percutaneous coronary revascularization with drug-eluting and bare metal stents in the United States. Circulation. 2013;127(18):1861-1869.

14. Charytan DM, Li S, Liu J, Herzog CA. Risks of death and end-stage renal disease after surgical compared with percutaneous coronary revascularization in elderly patients with chronic kidney disease. Circulation. 2012;126(11 Suppl 1):S164-S169.

15. Chan W, Ivanov J, Ko D, et al. Clinical outcomes of treatment by percutaneous coronary intervention versus coronary artery bypass graft surgery in patients with chronic kidney disease undergoing index revascularization in Ontario. Circ Cardiovasc Interv. 2015;8(1): e001973.

16. Manabe S, Shimokawa T, Fukui T, et al. Coronary artery bypass surgery versus percutaneous coronary artery intervention in patients on chronic hemodialysis: does a drug-eluting stent have an impact on clinical outcome? J Card Surg. 2009;24(3):234-239.

17. Krishnaswami A, McCulloch CE, Tawadrous M, et al. Coronary artery bypass grafting and percutaneous coronary intervention in patients with end-stage renal disease. Eur J Cardiothorac Surg. 2015;47(5): e193-e198. 


\section{Publish your work in this journal}

The International Journal of Nephrology and Renovascular Disease is an international, peer-reviewed open-access journal focusing on the pathophysiology of the kidney and vascular supply. Epidemiology, screening, diagnosis, and treatment interventions are covered as well as basic science, biochemical and immunological studies. The journal welcomes

original research, clinical studies, reviews \& evaluations, expert opinion and commentary, case reports and extended reports. The manuscript management system is completely online and includes a very quick and fair peerreview system, which is all easy to use. Visit http://www.dovepress.com/ testimonials.php to read real quotes from published authors.

Submit your manuscript here: http://www.dovepress.com/international-journal-of-nephrology-and-renovascular-disease-journal 